CLINICAL REPORT / REPORTE DE CASO

\title{
Coronavirus: First Report of a Sars-Cov-2 case In Ecuador. Are nutritional assessment and treatment helpful for a successful outcome?
}

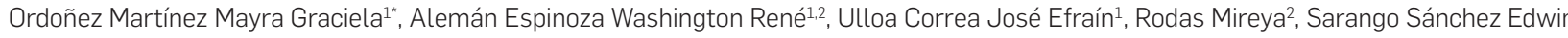 \\ Renato², Cucalón Gabriela ${ }^{3}$, Frias-Toral Evelyn ${ }^{4}$

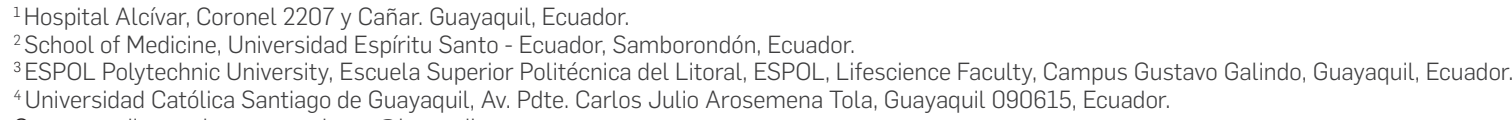

DOI. 10.21931/RB/2022.07.01.17

Abstract: Due to the globalized world in which we live, there are more excellent migratory movements and an increase in emerging diseases. During the past year, the significant impact of the coronavirus has been evident. It was previously designated 2019-nCoV and was identified as the cause of a cluster of pneumonia cases in Wuhan in late 2019. In February 2020, the World Health Organization (WHO) declared the COVID-19 coronavirus disease 2019, which causes severe acute respiratory syndrome (SARS-CoV-2). This outbreak's scope and ultimate effect remain unclear due to the fast spreading of new virus variants worldwide and the limitations to reaching a sufficient vaccination rate in low-income countries. We want to report the first case of COVID-19 in Ecuador and the lessons learned from its management.

Key words: Coronavirus, severe acute respiratory syndrome.

\section{Introduction}

A new variant for coronavirus emerged at the end of 2019 which was designated 2019-nCoV. It was identified as the cause of a cluster of pneumonia cases in Wuhan, China, and rapidly spread throughout China and worldwide. In February 2020, the World Health Organization (WHO) declared the COVID-19 disease $^{1}$, coronavirus disease 2019 , which causes severe acute respiratory syndrome (SARS-CoV-2) ${ }^{2}$. This outbreak's scope and ultimate effect are unclear as the situation rapidly evolves into new virus variants. We report the first case of COVID-19 in Ecuador to see retrospectively the actions taken to control its dissemination nationwide.

\section{CASE REPORT}

A 71-year-old woman was hospitalized in February 2020 for moderate-intensity headache, dry cough, non-quantified fever, progressive dyspnea, diaphoresis, and a feeling of restlessness. Something remarkable is that she came from Spain 8 days before. She had a medical history of hypertension and ischemic cerebrovascular event.

At her physical examination, bilateral sub-crepitant rales were found up to lung medium fields, leg edema (+++). Her temperature was 101.3 F, blood pressure 140/90 mmHg, heart rate 102 beats per minute, respiratory rate 28 per minute, oxygen saturation $88 \%$ at rest in room air, no reports of weight variations at admission or tobacco and alcohol consumption.

The patient was monitored with 02 supply by mask at 10 liters per minute, improving saturation to 93\%. Laboratory tests were done as presented in Table 1.
On admission, the chest X-ray showed bilateral basal and perihilar alveolar opacities with left basal confluent. Blood and urine culture tests and tests for Influenza A and B were negatives. Unfortunately, due to COVID 19 tests in Ecuador, it was impossible to perform the test from the patient's admission. The echocardiogram reported pulmonary hypertension with $59 \mathrm{mmHg}$.

High-resolution chest computerized tomography (CT) showed a left basal laminar pleural effusion, ground-glass opacities associated with thickening interlobular septa and bilateral bronchial dilatations (Figure. 1)

The patient was transferred to ICU with initial diagnoses of Acute Hypoxemic Respiratory Failure plus Severe Acute Pneumonia of the Community with a CURB-65 score: 2 (Mortality 6.8\% - intermediate risk) / SOFA score: 2 ( $\leq 33.3 \%$ mortality) / APACHE II score: 12 (predicted mortality rate 14.6\%) and Acute Pulmonary Edema. In the ICU, antibiotic treatment was started with Meropenem and Furosemide, and routine protocols were established. Also, the patient received enteral nutrition by nasogastric tube with a specialized formula low in carbohydrates, modified fat, and fiber (providing $1 \mathrm{cal} / \mathrm{mL}, 245$ $\mathrm{cal} / 250 \mathrm{~mL}$. Caloric Distribution: Protein 16.8\%, carbohydrates $34.2 \%$, fat $49 \%$ ) combined with a ready to use protein module made of soy (protein $6.8 \mathrm{gr}$ and carbohydrates $0.4 \mathrm{gr}$ ) diluted in 200 cc of water every 8 hours by intermittent infusion. The patient received non-invasive ventilation (NIV) due to 02 saturation that dropped to $90 \%$ and tachypnea to improve the ventilatory pattern.

Citation: Ordoñez Martínez M G, Alemán Espinoza W R, Ulloa Correa J E, Rodas M, Sarango Sánchez E R, Cucalón G, Frias-Toral E. Coronavirus: First Report of a Sars-Cov-2 case In Ecuador. Are nutritional assessment and treatment helpful for a successful outcome? Revis Bionatura 2022;7(1). 17. http://dx.doi.org/10.21931/RB/2022.07.01.17

Received: 9 July 2021 / Accepted: 31 October 2021 / Published: 15 February 2022

Publisher's Note: Bionatura stays neutral with regard to jurisdictional claims in published maps and institutional affiliations. 
The patient continued with no improvement, so treatment for typical and atypical germs was extended, along with antiviral coverage (Linezolid, Clarithromycin, Oseltamivir). In addition, a real-time multiplex RT-PCR of a nasal swab respiratory panel is performed, the result of which was negative for arbovirus, variants of coronavirus (229E, HKU1, NL63, OC43), adenovirus, influenza ( $\mathrm{A} / \mathrm{H1}, \mathrm{H} 3, \mathrm{~B})$, parainfluenza 1al4, respiratory syncytial virus, rhinovirus, enterovirus and atypical germs (Bordetella pertussis, Chlamydophila pneumoniae, Mycoplasma pneumoniae).

Between the 2nd and 3rd days of hospitalization, the patient remained in isolation in ICU, maintaining saturation but without radiological improvement. Finally, on the 4th day of hospitalization, the patient is anxious and does not tolerate NIV mask, her P02 decreases, leukocytes dropped to 9400, Neutrophils 85\% (Table 1). Due to her hypoxemia and irregular ventilatory pattern, the patient is intubated with pressure-controlled ventilation (PCV), under sedation, and analgesia with midazolam and fentanyl.

\begin{tabular}{|c|c|c|c|c|}
\hline Parameters/Date & $2 / 22 / 20$ & $2 / 24 / 20$ & $2 / 26 / 20$ & $2 / 28 / 20$ \\
\hline Leucocyte $\left(10^{\wedge} 3\right)$ & 17.96 & 16.7 & 9.40 & 10.7 \\
\hline Neutrophils & 15.79 & 15.0 & 6.71 & 9.17 \\
\hline Lymphocytes & 1.65 & 1.07 & 0.89 & 0.94 \\
\hline Platelets (k/ul) & 364 & 452 & 571 & 598 \\
\hline Hemoglobin (g/dl) & 11 & 10.9 & 10.8 & 9.9 \\
\hline Hematocrit & $32 \%$ & $30 \%$ & $30 \%$ & $28 \%$ \\
\hline Procalcitonin (ng/mL) & 0.34 & 0.5 & 0.6 & \\
\hline CRP (mg/dl) & 42 & -- & 29 & 28 \\
\hline D-dimer (ng/dl) & 2098 & & & \\
\hline \multicolumn{5}{|l|}{ Arterial Blood Gas } \\
\hline $\mathrm{pH}$ & 7.5 & 7.5 & 7.48 & 7.5 \\
\hline pCO2 & 27 & 36 & 42 & 41 \\
\hline pO2 & 126 & 129 & 61 & 112 \\
\hline SatO2 & $97 \%$ & $99 \%$ & $93 \%$ & $99 \%$ \\
\hline PAFI & 206 & 211 & & \\
\hline $\mathrm{HCO} 3(\mathrm{mmol} / \mathrm{L})$ & 21 & 28 & 31 & 32 \\
\hline
\end{tabular}

Table 1. Summary of Laboratory test.

On the 5th day of admission, a bronchoalveolar lavage $(B A L)$ is performed. Samples are sent to the National Institute of Public Health and Research (INSPI) to determine the RT-PCR of SARS-CoV-2 after coordination with the Ministry of Public health and reported positive. Then the patient was referred to a Contingency Hospital assigned for confirmed COVID-19 cases according to the Ecuadorian Ministry of Public Health (MPH) guidelines (Figure. 2).

\section{Discussion}

The coronavirus is still a public health emergency of international concern, with more than 152 million cases reported in 192 countries and regions ${ }^{3}$. Despite the great efforts to improve the vaccination rates in different nations, those with limited resources, as Ecuador, are dealing with this implacable virus and its new variants. The constant increasing COVID-19 cases, the attempts to return to normal economic activities, poverty, the Alfa, Beta, Gamma, Delta and other new variants are some of the factors that have severely affected the Ecuadorian health system. As a result, more hospital sections have been assigned to these patients; ICU beds, ventilators, $\mathrm{O} 2$ tanks, medicines, and all resources needed for their treatment are not enough. Unfortunately, this scenario is common in many countries in South America and other regions ${ }^{4}$. Hence, several governments have implemented quarantine measurements and flight restrictions, among other dispositions, to control this inexorable enemy.

Individuals exposed to COVID-19 cases must strictly follow the isolation and the guidelines defined by the MPH to control the virus spreading, especially since there are new, more virulent strains. Complications such as severe pneumonia, respiratory failure, acute respiratory distress syndrome (ARDS), and cardiac injury, including fatal outcomes, occur three to six days after suspected exposure.

As seen in the timeline (Figure. 2), 13 days after the onset of the symptoms, the patient was diagnosed with COVID-19. During that time, the "patient zero" made a series of contacts between family and friends in 2 very crowded cities from Ecuador. In that way, the primarily spread person-to-person through respiratory droplets was initiated. Notably, this person was among several that arrived in the country through the first 15 days of February. This time of the year is prevalent to receive travelers from Spain, Italy, and the United States.

This case report highlights the importance of clinicians obtaining a recent history of travel or exposure to COVID-19 positive cases in any patient presenting acute disease symptoms for early recognition. Also, to ensure proper identification and prompt isolation of patients at risk for 2019-nCoV infection to help reduce further transmission. As for the community, people should be advised to practice social distance, wear a medical mask, diligent hand washing, respiratory hygiene to contain their respiratory secretions, and seek medical attention in case of suspicion ${ }^{5}$.

Nutritional assessment and treatment are still not part of the routine protocols in many institutions worldwide, as demonstrated by the nutritional papers ${ }^{6}$. After all these months, where more knowledge around COVID-19 has been developed, the profound impact of nutritional status on these patients has been shown. Many researchers have highlighted the direct connection between an altered nutritional status with prolonged stay, more significant admission in the ICU, and higher mortality rates ${ }^{7}$. In Ecuador, a multidisciplinary group elaborated the guidelines for assessment and treatment of COVID-19 cases, and throughout the guideline, it has been emphasized the fundamental role of the nutritional status for a patient's prognosis ${ }^{8}$. This nutritional approach also applies to cancer patients who have been severely affected by this pandemic, as it was published by Rodriguez et al. ${ }^{9}$.

While the governments are making exceptional attempts to reach herd immunization, it remains imperative not to forget the fundamental things to prevent COVID-19 infection and treat them through a nutritional assessment. It can be determined if there is any degree of malnutrition as recommended by the ESPEN guidelines to do the needed corrections to improve the patient's prognosis ${ }^{10}$. Remarkably, these guidelines were developed in collaboration with WHO representatives, one of the leading institutions about this disease. 


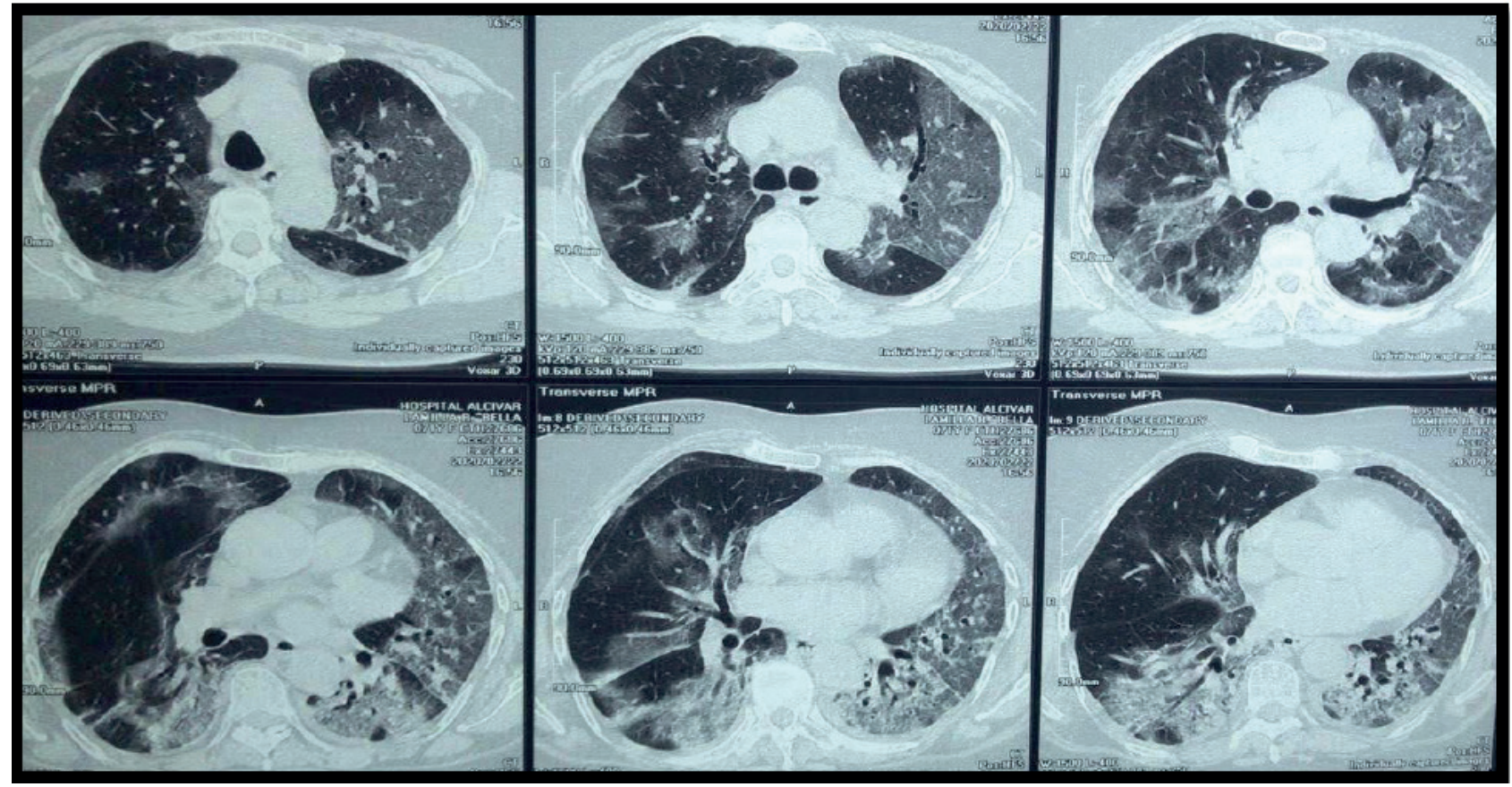

Figure 1. Chest CT scan showing left basal laminar pleural effusion, ground-glass opacities associated with thickening interlobular septa and bilateral bronchial dilatations.

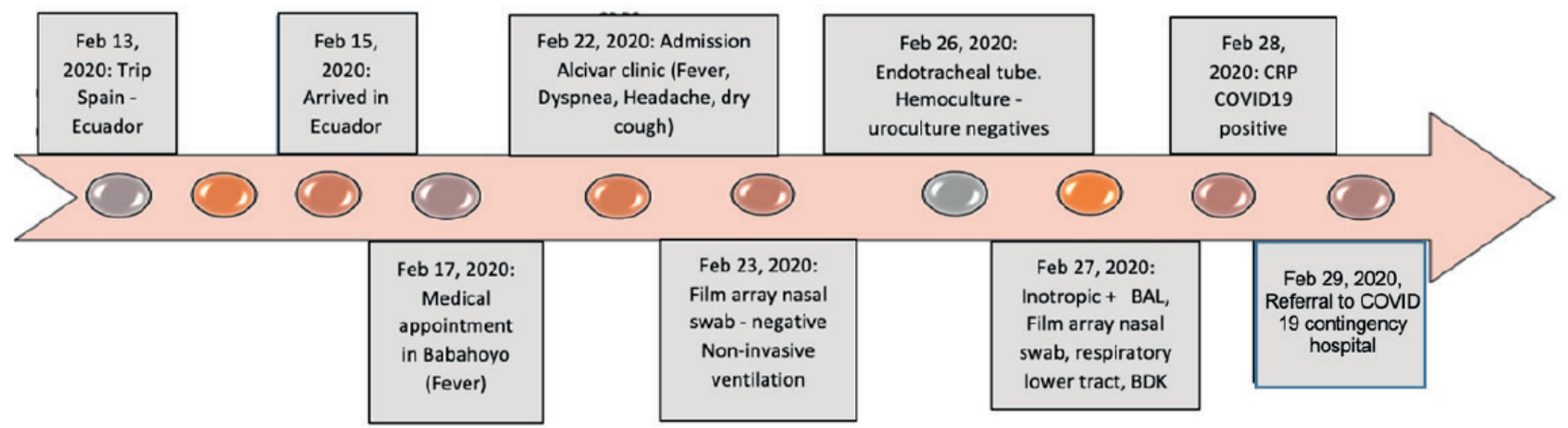

Figure 2. Timeline of significant events in the reported case. BAL: bronchoalveolar lavage.

\section{Conclusions}

We hope this case can emphasize the relevance of initiating an early diagnosis and installing correct protocols to control COVID-19 dissemination. Also, it stresses that nutritional assessment and intervention are essential pillars to prevent and contribute to the integral management of these patients, especially in countries where the vaccination rates, due to several reasons, are not as fast as the number of positive cases.

\section{Statement of authorship}

All authors contributed equally to this case-report communication.

\section{Funding Source}

None

\section{Statement of Ethics}

The patient provided both verbal and written informed consent to publish the case, including the publication of images.

\section{Acknowledgments}

None

\section{Bibliographic references}

1. Cucinotta D, Vanelli M. WHO Declares COVID-19 a Pandemic. Acta Biomed. 2020 Mar 19;91(1):157-160. doi: 10.23750/abm. v91i1.9397. PMID: 32191675; PMCID: PMC7569573.

2. Coronaviridae Study Group of the International Committee on Taxonomy of Viruses. The species Severe acute respiratory syndrome-related coronavirus: classifying 2019-nCoV and naming it SARS-CoV-2. Nat Microbiol. 2020 Apr;5(4):536-544. doi: 10.1038/ s41564-020-0695-z. Epub 2020 Mar 2. PMID: 32123347; PMCID: PMC7095448.

3. Dong E, Du H, Gardner L. An interactive web-based dashboard to track COVID-19 in real time. Lancet Inf Dis. 20(5):533-534. doi: 10.1016/S1473-3099(20)30120-1

4. Alava JJ, Guevara A. A critical narrative of Ecuador's preparedness and response to the COVID-19 pandemic. Public Health Pract (Oxf). 2021 Nov;2:100127. doi: 10.1016/j.puhip.2021.100127. Epub 2021 Apr 23. PMID: 33907741; PMCID: PMC8062908.

\section{Declaration of Competing Interest}

We have no competing interests to disclose. 
5. Cuéllar L, Torres I, Romero-Severson E, Mahesh R, Ortega N Pungitore $S$, et al. Excess deaths reveal the true spatial, temporal, and demographic impact of COVID-19 on mortality in Ecuador. medRxiv [Preprint]. 2021 Mar 1:2021.02.25.21252481. doi: 10.1101/2021.02.25.21252481. PMID: 33688690; PMCID: PMC7941667.

6. Cereda E, Klersy C, Hiesmayr M, Schindler K, Singer P, Laviano A, et al. NutritionDay Survey Collaborators. Body mass index age and in-hospital mortality: The NutritionDay multinational survey. Clin Nutr. 2017 Jun;36(3):839-847. doi: 10.1016/j. clnu.2016.05.001. Epub 2016 May 15. PMID: 27236599.

7. Yu Y, Ye J, Chen M, Jiang C, Lin W, Lu Y, et al. Malnutrition Prolongs the Hospitalization of Patients with COVID-19 Infection: A Clinical Epidemiological Analysis. J Nutr Health Aging. 2021;25(3):369373. doi: 10.1007/s12603-020-1541-y. PMID: 33575730; PMCID: PMC7709472.

8. Ministry of Health. Consenso interino multidisciplinario informado en La evidencia sobre El tratamiento de COVID19. Ecuador: Government of Ecuador, 2020.
9. Rodriguez D, Guerrero M, Maldonado M, Herrera S, Frías E, Santana, S. Recomendaciones en el tratamiento nutricional para pacientes oncológicos ante covid-19. J Health Med Sci. 2020; 6(4):303-314.

10.Barazzoni R, Bischoff SC, Breda J, Wickramasinghe K, Krznaric Z, Nitzan D, et al; endorsed by the ESPEN Council. ESPEN expert statements and practical guidance for nutritional management of individuals with SARS-CoV-2 infection. Clin Nutr. 2020 Jun;39(6):1631-1638. doi: 10.1016/j.clnu.2020.03.022. Epub 2020 Mar 31. PMID: 32305181; PMCID: PMC7138149. 